\title{
ROS sets the stage for macrophage differentiation
}

\author{
Cell Research (2013) 23:984-985. doi:10.1038/cr.2013.88; published online 9 July 2013
}

While M1 macrophages are highly pro-inflammatory and microbicidal, M2 macrophages and the related tumor associated macrophages (TAMs) regulate tissue remodeling and angiogenesis and can display immunomodulatory activity. In July issue of Cell Research, Zhang et al. show that ROS production, critical for the activation and functions of M1 macrophages, is necessary for the differentiation of M2 macrophages and TAMs, and that antioxidant therapy blocks TAM differentiation and tumorigenesis in mouse models of cancer.

Macrophages are key orchestrators in both the initiation and resolution stages of inflammation, and function as sentinel cells that maintain homeostasis and protect against infection. They are activated by many stimuli including pathogen-associated molecular patterns (PAMPs), endogenous danger-associated molecular patterns (DAMPs), and cytokines found in the tissue microenvironment [1]. During their activation, macrophages can polarize to pro-inflammatory or anti-inflammatory states with distinct phenotypes and physiological responses - the classical pro-inflammatory M1 state induced by LPS and interferon- $\gamma$ (IFN- $\gamma$ ) and the "alternative" M2 state triggered by IL-4 and IL-13 [2]. The M1 state is characterized by increased expression of pro-inflammatory cytokines as well as microbicidal activity, while M2 macrophages upregulate the anti-inflammatory cytokine IL-10 and participate in tissue remodeling, wound repair, and host defense against large parasites.

M2-like macrophage polarization is of particular pathophysiological consequence in the setting of cancer. Early in tumor development, monocytes are recruited by tumor and stromal cell-derived chemokines to take up residence at the tumor site, where they differentiate into macrophages in response to MCSF produced by tumor cells. Such tumor-associated macrophages (TAMs) facilitate multiple steps in tumorigenesis, including promotion of tumor cell proliferation and resistance to apoptosis as well as secretion of pro-angiogenic factors and proteolytic enzymes that aid tumor cell metastasis. TAMs also display some immunosuppressive features, such as IL-10 and TGF- $\beta$ production and poor antigen presentation, which conspire to prevent tumor cell killing by infiltrating $\mathrm{T}$ cells. Thus, the characteristics most critical for the tumor-promoting profile of TAMs bear semblance to the M2 phenotype. Although the details of such M2 polarization are not well characterized, IL-4 produced by T-cells in the tumor, as well as other tumor-derived factors, may be critical [3].

In July issue of Cell Research, a study by Zhang et al. [4] provides new insights into control of macrophage differentiation and activation. In particular, the authors show that ROS production is important in M2 but not M1 macrophage differentiation. Their experimental protocol is to treat monocytes for 6 days with M-CSF or GM-CSF to induce differentiation to macrophages, followed by polarization with IL-4 (M2 state) or LPS and IFN- $\gamma$ (M1 state). Interestingly, pre-treating monocytes with the antioxidant butylated hydroxyanisole (BHA) prior to differentiation inhibits M2 but not M1 polarization, as indicated by analysis of macrophage differentiation markers and M1/M2 polarization markers. The authors attribute this to the effects of BHA, i.e., block of ROS production, in inhibiting ERK activation during macrophage differentiation, consistent with previous reports implicating a role for ROS as well as MAP kinases in macrophage differentiation [5]. Furthermore, LPS and IFN- $\gamma$ but not IL-4 stimulation can "rescue" ERK activation, perhaps in a manner dependent on ROS production, thus explaining why M2 but not M1 polarization is impaired by antioxidant treatment (Figure 1).

As the M2-like properties of TAMs are thought to promote tumorigenesis, Zhang et al. go on to investigate the consequences of BHA administration in mouse models of cancer. They demonstrate that in vivo treatment of BHA can attenuate cancer initiation, progression, and metastasis in multiple models. As ROS can promote tumor cell proliferation, survival, and DNA damage, BHA could be acting directly on the tumor cells to prevent growth and metastasis [6]. However, BHA had no effects on the proliferation of three tumor cell lines in vitro. The authors propose that TAM differentiation may be a critical target, as BHA administration reduced TAM numbers as well as levels of TAM markers. Moreover, in at least one of the models, BHA administration was ineffective when macrophages were depleted by clodronate injection.

Collectively, the findings of Zhang et al. are intriguing for several reasons. First, ROS production is usually associated with the activation and functions of M1 rather than M2 macrophages. 


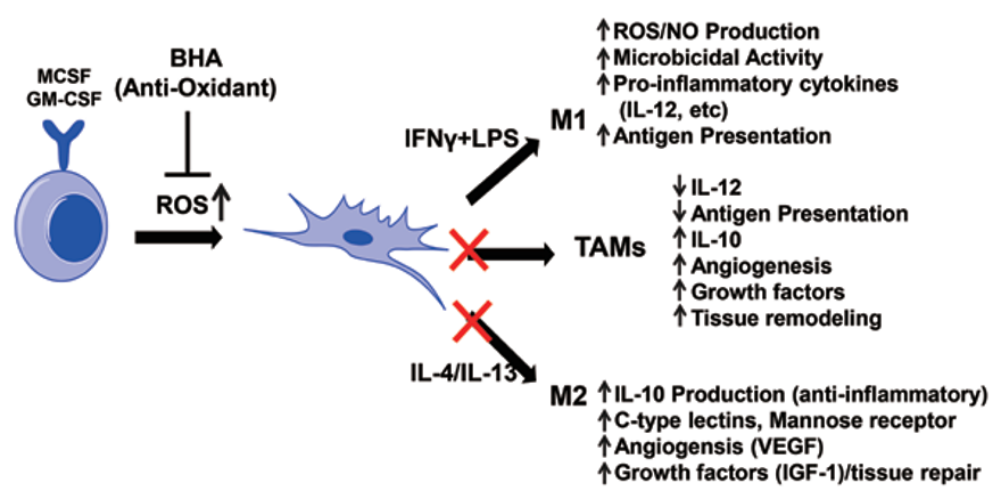

Figure 1 M1 macrophages are highly pro-inflammatory and microbicidal and are polarized by treatment with LPS+IFN $\gamma$, while M2 macrophages mediate tissue repair, angiogenesis and immunomodulation. Tumor associated macrophages (TAMs), which are M2-like, are associated with worsened clinical prognosis in many cancers and are thought to be skewed by a combination of tumor-derived factors and other cytokines present in the tumor microenvironment. ROS production increases during M-CSF- or GM-CSF-induced macrophage differentiation from monocytes, and the antioxidant BHA specifically inhibits M2 and TAM polarization. LPS+IFN $\gamma$ treatment is able to overcome the effects of BHA to induce normal M1 polarization, revealing a specific role for ROS in macrophage polarization.

ROS production downstream of LPS signaling mediates production of proinflammatory cytokines (in part through MAP kinase activation). ROS and nitric oxide (NO) production by NADPH oxidase and iNOS, respectively, as well as mROS upregulation are key to the antimicrobial activity of M1 macrophages [7]. Indeed NO production can inhibit oxidative metabolism, pivotal to the survival and function of M2 macrophages [8]. Thus ROS production may be important in M1 activation and function while the requirement for ROS in M2 differentiation may be most critical during MCSF-mediated differentiation rather than IL-4-triggered polarization. Future studies to better understand the role of ROS production in macrophage differentiation and activation may be informative. Second, it would be interesting to further probe the effects of BHA in inhibiting tumorigenesis. The authors' in vitro studies suggest inhibition of TAM differentiation as one underlying mechanism, but one can envision additional possibilities. At least in some cancers, tumor cells and other immune cells in the microenvironment produce ROS that promote inflammation [9], thus contributing to tumorigenesis. mROS has been linked to activation of HIF $1 \alpha$, which can facilitate angiogenesis and metastasis. Indeed, it is worth pointing out that ROS can regulate many cellular processes, some of which have already been alluded to, including signal transduction (e.g., downstream of growth factor receptors and innate immune signaling pathways as well as MAP kinase activation), redox signaling, autophagy, and respiratory burst and other antimicrobial activities [10]. Thus it is likely that other cellular processes perturbed by antioxidant treatment contribute to the effects of BHA in reducing tumorigenesis.

Finally, the study by Zhang et al. suggests that treatment with BHA or perhaps other antioxidants could be considered in therapeutic control of cancer. Indeed, there is tremendous interest in the clinical use of antioxidants for treating many diseases. Given the pleiotropic activities of ROS mentioned above, it would be important to better understand the molecular pathways by which antioxidants exert their effects.

Anthony Covarrubias ${ }^{1}$, Vanessa Byles ${ }^{1}$, Tiffany Horng ${ }^{1}$

${ }^{1}$ Department of Genetics and Complex Diseases, Harvard School of Public Health, Boston, MA 02115, USA

Correspondence: Tiffany Horng

E-mail: thorng@hsph.harvard.edu

\section{References}

1 Rakoff-Nahoum S, Medzhitov R. Nat Rev Cancer 2009; 9:57-63.

2 Gordon S, Taylor PR. Nat Rev Immunol 2005; 5:953-964.

3 Allavena P, Mantovani A. Clin Exp Immunol 2012; 167:195-205.

4 Zhang Y, Choksi S, Chen K, et al. Cell Res 2013; 23:898-914.

5 Sardina JL, Lopez-Ruano G, SanchezAbarca LI, et al. Cell Death Differ 2010; 17:1842-1854.

6 Storz P. Front Biosci 2005; 10:1881-1896.

7 Reuter S, Gupta SC, Chaturvedi MM, et al. Free Radic Biol Med 2010; 49:1603-1616.

8 O'Neill LA, Hardie DG. Nature 2013; 493:346-355.

9 Grivennikov SI, Greten FR, Karin M. Cell 2010; 140:883-899.

10 Finkel T. J Cell Biol 2011; 194:7-15. 